\title{
CONDENSAÇÃO ALDÓLICA DE FURFURAL E ACETONA CATALISADA POR BASES ORGÂNICAS NITROGENADAS: UM ESTUDO PRELIMINAR DE DESEMPENHO CATALÍTICO VISANDO A PRODUÇ̃̃O DE BIOQUEROSENE DE AVIAÇÃO
}

\author{
Vinícius W. Faria, ${ }^{a}$ Guilherme C. Almeida ${ }^{\mathrm{b}}$ e Cláudio J. A. Mota ${ }^{\mathrm{a}, \mathrm{b}, \mathrm{c}, *}$ \\ ${ }^{a}$ Escola de Química, Universidade Federal do Rio de Janeiro, 21941-901 Rio de Janeiro - RJ, Brasil \\ 'Instituto de Química, Universidade Federal do Rio de Janeiro, 21941-901 Rio de Janeiro - RJ, Brasil \\ 'INCT Energia e Ambiente, Universidade Federal do Rio de Janeiro 21941-909 Rio de Janeiro - RJ, Brasil
}

Recebido em 17/10/2017; aceito em 05/03/2018; publicado na web em 04/04/2018

\begin{abstract}
ALDOL CONDENSATION OF FURFURAL WITH ACETONE CATALYZED BY NITROGENATED ORGANIC BASES: A PRELIMINARY STUDY OF THE CATALYTIC PERFORMANCE TOWARD THE PRODUCTION OF BIOJET FUEL. The aldol condensation between furfural and acetone was studied in the presence of organic bases, such as Piperazine (PIP), 3-Aminopropyltriethoxysilane (APTES) and 1,5,7-Triazabicyclo [4.4.0] dec-5-ene (TBD), as basic catalysts. The latter base showed the highest conversion rates of furfural and high selectivity to the single-condensation product 4-(2-Furyl)-3-buten-2-on (F-Ac). These data are consistent with the greater basicity of TBD in relation to the other amines. This study was carried out aiming at the future anchoring of the bases in inorganic supports for the production of heterogeneous catalysts.
\end{abstract}

Keywords: furfural; acetone; aldol condensation; TBD.

\section{INTRODUÇÃO}

Novas alternativas tecnológicas sustentáveis têm sido desenvolvidas no sentido de buscar novos caminhos para substituir os combustíveis de origem fóssil. A produção de biocombustíveis é uma das formas de se gerar energia a partir de recursos renováveis. ${ }^{1-5} \mathrm{O}$ bioquerosene de aviação é um biocombustível inovador ainda pouco explorado, porém, com grande potencial devido à possibilidade de uso de matéria-prima oriunda de biomassa. ${ }^{6,7}$

O processo de produção de biocombustível envolve não somente a escolha da matéria-prima, mas também a escolha de uma rota química para geração do produto. Quatro rotas tecnológicas de produção de bioquerosene vêm recebendo destaque: ${ }^{8-11}$

(a) transesterificação/descarbonilação/hidrogenação de óleos vegetais e gorduras;

(b) síntese de Fischer-Tropsch a partir de biomassa (processos BTL);

(c) hidroprocessamento de óleo vegetal;

(d) condensação aldólica e hidrogenação de furfural e derivados.

As rotas são interessantes sob o ponto de vista sustentável. No entanto, possuem grandes desafios, uma vez que exploram um campo ainda não economicamente rentável. As rotas (a) e (c) têm como fonte de biomassa os triglicerídeos, já a última rota tem como fonte a decomposição de açúcares presentes em biomassa lignocelulósica, podendo ter alto potencial para produção de biocombustíveis. ${ }^{10-13} \mathrm{~A}$ hidrólise de celulose e hemicelulose, seguida da degradação ácida da glicose e xilose produzidas formam o 5-hidroximetilfurfural e o furfural, respectivamente. A condensação aldólica entre o furfural e acetona pode formar compostos oxigenados de maior cadeia que, uma vez hidrogenados, podem produzir alcanos na faixa de 8 a 16 átomos de carbono, ideal para a produção de querosene de aviação. A reação envolve a formação do intermediário 4-(2-Furil)-4-hidroxi-2-butanona (F-OH) que, na sequência, desidrata formando o produto insaturado 4-(2-Furil)-3-buten2-ona (F-Ac). Este produto pode reagir com uma segunda molécula

*e-mail: cmota@iq.ufrj.br de furfural e formar o 1,4-pentadien-3-ona-1,5-di-2-furanil (F-Ac-F) ${ }^{14}$ conforme o esquema reacional mostrado na Figura 1.

A presença de catalisadores básicos na condensação aldólica entre furfural e a acetona é imprescindível. ${ }^{15,16}$ Muitos processos químicos industriais utilizam catalisadores em pelo menos uma das etapas. Um catalisador deve apresentar altas taxas de conversão e seletividade aos produtos desejados. ${ }^{17}$ Dumesic e colaboradores publicaram ${ }^{18} \mathrm{um}$ trabalho referente à produção de n-alcanos líquidos por uma rota química baseada em carboidratos de biomassa ligninocelulósica. A combinação sequencial de reações de hidrólise/desidratação de polissacarídeos, condensação aldólica e hidrogenação, forma uma rota química atraente, recentemente aplicada na formulação de combustíveis automotivos. A desidratação foi realizada por catalisadores ácidos, a condensação aldólica por catalisadores sólidos básicos e a desidratação/hidrogenação por catalisadores de metais nobres suportados. A principal ideia é a utilização de um reator catalítico de quatro fases com entradas para cada componente: reagentes orgânicos em meio aquoso, hexadecano, gás hidrogênio e catalisador. O hexadecano torna a fase orgânica mais hidrofóbica removendo as espécies hidrofóbicas (produtos) do catalisador antes de haver a deposição de coque no catalisador.

West, Dumesic e colaboradores empregaram um sistema bifásico, utilizando tetraidrofurano (THF), com objetivo de separar os produtos solúveis no meio orgânico e insolúveis em água. O cloreto de sódio é usado para diminuir a miscibilidade entre as fases orgânica e aquosa. ${ }^{11}$ No entanto, diante do uso do solvente orgânico e quantidade de água no processo, o sistema bifásico não parece ser a melhor alternativa para produção dos n-alcanos lineares. Constata-se, também, a formação de ácidos orgânicos que podem neutralizar o catalisador básico. ${ }^{19}$ Catalisadores óxidos metálicos e hidrotalcitas em meio aquoso foram, também, testados na reação. ${ }^{20,21}$ Há estudos sobre a aplicação de catalisadores zeolíticos em testes catalíticos na reação de condensação aldólica. ${ }^{22,23}$

Esta rota foi testada com três catalisadores diferentes usados em cada etapa. Inicialmente, os carboidratos foram hidrolisados na presença de $\mathrm{HCl}$ e transformados em hemicelulose e oligômeros de xilose. Na sequência, os açúcares foram desidratados para formar o furfural. 


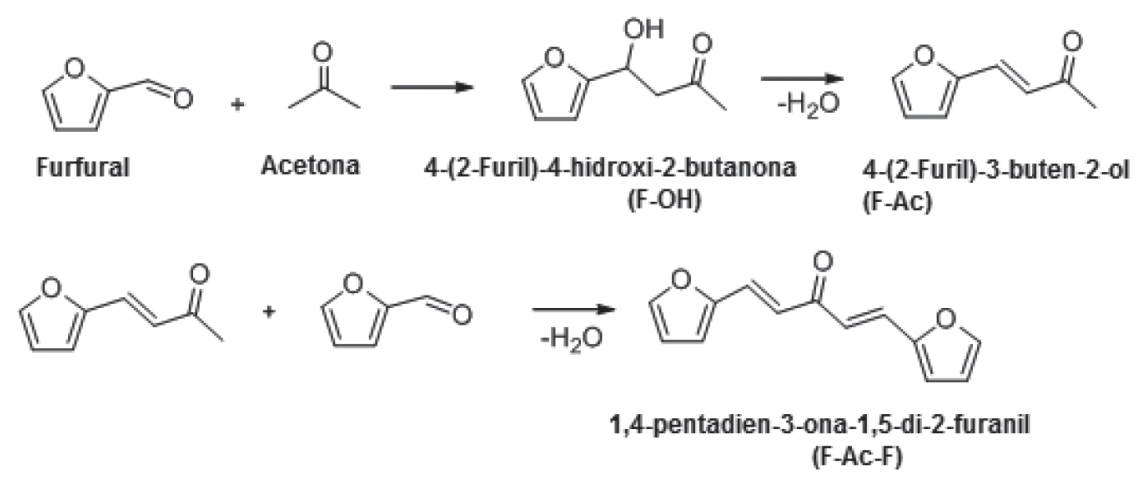

Figura 1. Condensação aldólica entre a acetona e o furfural

A reação de condensação aldólica entre furfural e acetona, seguida da hidrogenação dos intermediários formados, produziu n-alcanos. A reação de condensação aldólica foi catalisada por $\mathrm{NaOH}$ levando a excelentes rendimentos. Os catalisadores metálicos suportados utilizados nas reações de hidrogenação foram $\mathrm{Pd} / \mathrm{Al}_{2} \mathrm{O}_{3}$ e $\mathrm{Pt} / \mathrm{NbOPO}_{4}{ }^{11}$

Os compostos orgânicos com funções aminas possuem características básicas de Lewis e de Bronsted. O 1,5,7-Triazabiciclo[4.4.0] dec-5-eno (TBD) é uma base forte com valor de pKa do ácido conjugado igual 26. ${ }^{24} \mathrm{O}$ TBD tem sido usado na catálise de algumas reações orgânicas como, por exemplo, na reação de Henry, ${ }^{25}$ na reação de Michael, ${ }^{26}$ reação de Horner-Wadsworth-Emmos, ${ }^{27}$ Claisen-Schimidt ${ }^{28}$ e entre benzaldeído e 1-metoxi-2-metil-1-trimetil siloxipropeno. ${ }^{29}$ Nosso grupo empregou esta base ancorada em sílica mesoporosa na transesterificação de óleos vegetais para produção de biodiesel, obtendo excelentes rendimentos..$^{30} 3$-Aminopropil trietoxisilano (APTES) possui um grupoamino primário, enquanto que a piperazina tem estrutura cíclica com a presença de dois grupos aminos secundários. Elas possuem valores de pKa do ácido conjugado igual a 10,5 e 9,8, respectivamente. Ambas já foram aplicadas em reações de condensação de Knoevenage ${ }^{31-33}$ e também na captura de dióxido de carbono. ${ }^{33,34}$ A Figura 2 mostra as estruturas químicas dos compostos orgânicos.

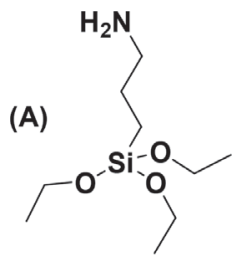

(B)<smiles>C1CNCCN1</smiles>

(C)

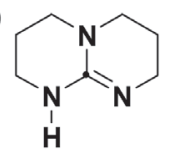

Figura 2. Estrutura química de compostos amino-orgânicos (a) APTES, (b PIP e (c) TBD

No presente trabalho, propõe-se empregar compostos orgânicos básicos com grupamentos funcionais amino primário, secundário e terciário, com diferentes basicidades, visando um estudo preliminar catalítico com aplicação na reação de condensação aldólica entre furfural e acetona. Os compostos testados foram a Piperazina (PIP), o 3-Aminopropiltrietoxisilano (APTES) e o 1,5,7-Triazabiciclo[4.4.0] dec-5-eno (TBD). A ideia é poder verificar a viabilidade de ancoragem destas bases em suportes inorgânicos visando a heterogeneização posterior do sistema catalítico.

\section{PARTE EXPERIMENTAL}

\section{Reagentes e solventes}

Furfural e acetona foram adquiridos da Sigma-Aldrich, em grau analítico, bem como as bases orgânicas piperazina (PIP),
3-Aminopropiltrietoxisilano (APTES) e 1,5,7-Triazabiciclo[4.4.0] dec-5-eno (TBD).

\section{Avaliação catalítica}

A avaliação catalítica foi realizada variando-se os seguintes parâmetros: tipo e quantidade de catalisador (TBD, APTES e PIP), temperatura de reação e razão molar Acetona/Furfural. A quantidade de furfural nas reações foi de $1 \mathrm{~mL}$. Assim, para razão molar 10:1 acetona: furfural a quantidade de acetona foi de $9 \mathrm{~mL}$. Em todos os testes de avaliação catalítica a agitação no reator tipo Parr foi mantida em $900 \mathrm{rpm}$. Após o final de cada experimento foi retirada uma alíquota do meio reacional e solubilizada em acetato de etila, sendo então analisada em equipamento de cromatografia gasosa acoplada a espectrômetro de massas. A coluna cromatográfica foi do tipo HP-5 (5\% Fenil, 95\% Dimetilpolisiloxano) com comprimento de $30 \mathrm{~m}$, espessura de filme igual a $0,25 \mu \mathrm{m}$ e diâmetro interno de $0,25 \mathrm{~mm}$. As condições de análise foram: temperatura inicial de forno igual a $50{ }^{\circ} \mathrm{C}$, tempo inicial de 1,5 min e temperatura máxima em $250{ }^{\circ} \mathrm{C}$. A taxa de aquecimento é igual a $15^{\circ} \mathrm{C} \mathrm{min}{ }^{-1}$, modo split 100:1. A conversão de furfural, seletividade ao F-Ac e frequência de turnover estão descritas pelas equações 1, 2, 3 e 4, tendo sido obtidas a partir das análises cromatográficas através de curvas de calibração.

Conversão Furfural $(\%)=\frac{\text { mols furfural início }- \text { mols furfural final }}{\text { mols furfural início }}$

Seletividade F-AC $(\%)=\frac{\text { mols } \mathrm{F}-\mathrm{Ac}}{\text { mols Furfural início }- \text { mols furfural final }}$

$$
\begin{gathered}
\text { Frequência de turnover }=\frac{\text { mols } \mathrm{F}-\mathrm{Ac}}{\text { mols TBD.tempo }} \\
\text { Rendimento }(\%)=\frac{\text { mols F-Ac }}{\text { mols furfural início }}
\end{gathered}
$$

Na primeira série de testes catalíticos avaliou-se a atividade dos catalisadores TBD, APTES e PIP frente à reação de condensação aldólica entre a acetona e furfural a $50{ }^{\circ} \mathrm{C}$, durante $7 \mathrm{~h}$, com $1,4 \mathrm{~mol} \%$ de catalisador/mol de furfural e razão molar 10:1 acetona/furfural. Alguns efeitos de variáveis independentes reacionais foram estudados ao melhor catalisador avaliado: temperatura, quantidade de catalisador e razão molar acetona/furfural.

Na segunda série de testes de avaliação catalítica, o catalisador 1,5,7-Triazabiciclo[4.0.0]dec-5-eno (TBD) foi testado frente à condensação aldólica entre a acetona e o furfural, 1,4 mol\% catalisador/ mol furfural em todos os experimentos. Cada experimento foi avaliado em diferentes temperaturas: $25^{\circ} \mathrm{C}, 50{ }^{\circ} \mathrm{C}, 75^{\circ} \mathrm{C}$ e $100^{\circ} \mathrm{C}$. O tempo 
reacional foi igual a $7 \mathrm{~h}$ e a razão molar 10:1 acetona/furfural.

Na terceira série de testes de avaliação do catalisador, todos os experimentos foram feitos à temperatura de $50{ }^{\circ} \mathrm{C}$, variando-se a quantidade de catalisador 0,$7 ; 1,4 ; 3$ e 6 mol\% de catalisador/mol de furfural. $\mathrm{O}$ tempo reacional experimental foi de $7 \mathrm{~h}$ e a razão molar acetona /furfural foi de 10:1 em todos os experimentos da série.

$\mathrm{Na}$ quarta série de testes de avaliação catalítica a temperatura reacional foi mantida a $50{ }^{\circ} \mathrm{C}$ durante $6 \mathrm{~h}$ e variou-se a razão molar acetona/furfural. Foram testadas as razões molares acetona/furfural de $1: 2,1: 1,5: 1,10: 1$ e 15:1. A quantidade de catalisador 1,4 mol\% de catalisador/mol furfural foi mantida constante em todos os testes.

\section{RESULTADOS E DISCUSSÃO}

\section{Comparação de catalisadores à base amino-orgânica}

Na primeira série de testes catalíticos os três catalisadores, TBD, APTES e PIP foram comparados na reação de condensação aldólica entre furfural e acetona. A Figura 3 mostra a conversão de furfural com o tempo. Nota-se a maior atividade catalítica do TBD, que promoveu $100 \%$ de conversão após cerca de 7 horas de reação. Neste mesmo tempo, o APTES converteu pouco mais de $47 \%$ de furfural e a PIP apenas cerca de $15 \%$. A justificativa pode ser relacionada à basicidade das aminas. $\mathrm{O}$ pKa dos ácidos conjugados do TBD é igual a 26, enquanto que o pKa do APTES e PIP são 10,5 e 9,8 , respectivamente. Nestes testes catalíticos observou-se somente o intermediário F-OH e o produto insaturado F-Ac. Inicialmente, a base remove o hidrogênio alfa da acetona resultando em espécie rica em elétrons denominada enolato (carbânion). Este íon é estabilizado pela deslocalização de carga por ressonância em direção ao átomo de oxigênio. A seguir, o carbânion ataca a carbonila do furfural formando uma nova ligação C-C. Após transferência de próton é formado o intermediário F-OH. Na sequência, pode ocorrer nova abstração

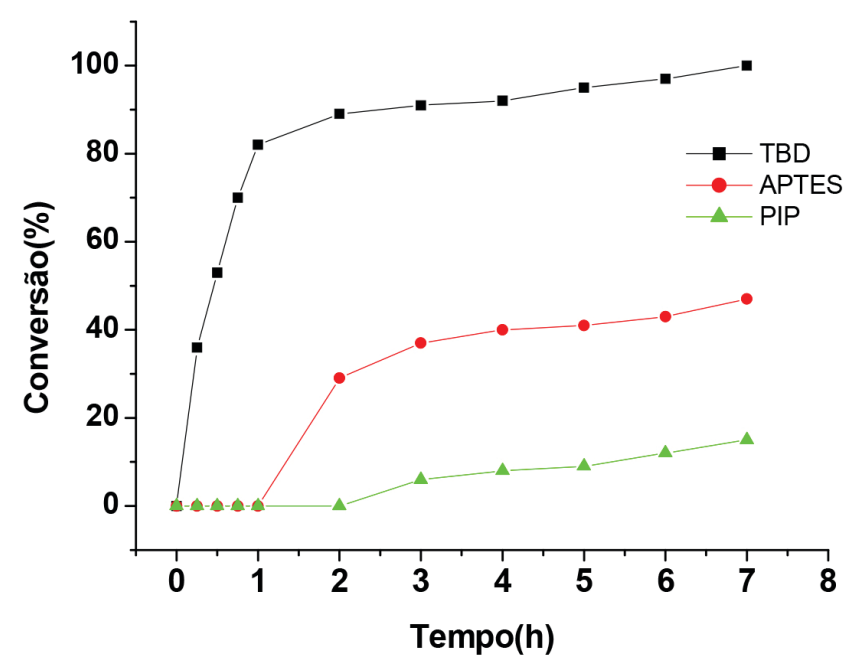

Figura 3. Conversão do furfural com o tempo de reação. Condições reacionais: $50{ }^{\circ} \mathrm{C}, 1,4 \mathrm{~mol} \%$ de catalisador/mol furfural, razão molar acetonal furfural 10:1 do próton ácido adjacente à carbonila para gerar um enolato, o qual pode, na sequência, eliminar grupamento $\mathrm{OH}^{-}$para gerar o produto insaturado 4-(2-Furil)-3-buteno-2-ona (F-Ac).

A Tabela 1 mostra os resultados de conversão e seletividade para os catalisadores a $50{ }^{\circ} \mathrm{C}$ e $2 \mathrm{~h}$ de reação. A frequência de turnover [F.T.] foi calculada como parâmetro de atividade catalítica. Nota-se que o catalisador TBD teve maior frequência de turnover, logo maior atividade catalítica quando comparado aos catalisadores APTES e PIP. A seletividade ao F-Ac também foi maior com o catalisador TBD, corroborando a hipótese de sua maior basicidade ser a responsável pelo melhor desempenho catalítico, tanto em termos de conversão como de seletividade ao produto desejado.

Os testes catalíticos seguintes foram realizados com o catalisador $\mathrm{TBD}$, já que foi o que promoveu o melhor desempenho nos testes comparativos. Os experimentos foram realizados com objetivo de investigar a influência das variáveis independentes na conversão de furfural e seletividade ao produto F-Ac. Os efeitos estudados foram a temperatura, quantidade de catalisador ( $\mathrm{mol} \%$ catalisador $/ \mathrm{mol} \mathrm{de}$ furfural) e razão molar acetona/furfural. O estudo destes efeitos busca encontrar uma faixa operacional de testes experimentais de reação para avaliar a viabilidade do uso de TBD ancorado em suportes inorgânicos utilizados como catalisadores heterogêneos.

\section{Efeito da temperatura na atividade catalítica do TBD}

O efeito da temperatura de reação foi estudado devido à sua importância na cinética de reação. Além disso, altas temperaturas podem favorecer a degradação do furfural, podendo formar co-produtos orgânicos ácidos, que podem neutralizar a base e limitar a formação de produtos da condensação. A Figura 4 mostra o perfil da conversão de furfural e seletividade ao produto F-Ac ao longo do tempo na faixa de temperatura investigada. Foram realizados quatro testes visando a comparação entre diferentes temperaturas $25^{\circ} \mathrm{C}, 50{ }^{\circ} \mathrm{C}, 75^{\circ} \mathrm{C}$ e 100 ${ }^{\circ} \mathrm{C}$, usando $1,4 \mathrm{~mol} \%$ de catalisador TBD/mol de furfural e $7 \mathrm{~h}$ de reação. A conversão do furfural variou de $60 \%$ a $100 \%$ com o aumento da temperatura entre $25^{\circ} \mathrm{C}$ e $100{ }^{\circ} \mathrm{C}$. Nota-se que os resultados a 75 e $100{ }^{\circ} \mathrm{C}$ não diferem significativamente, com $100 \%$ de conversão na primeira hora de reação. Em todos os experimentos houve somente a formação do intermediário F-OH e do produto F-Ac. A $25^{\circ} \mathrm{C}$, ainda com baixa conversão de furfural, observa-se uma maior seletividade ao intermediário F-OH e baixa seletividade ao produto F-Ac. O teste a $50{ }^{\circ} \mathrm{C}$ já mostra maiores conversões de furfural e diminuição da seletividade ao intermediário $\mathrm{F}-\mathrm{OH}$, com prevalência do produto $\mathrm{F}$-Ac. Nas temperaturas mais altas, a conversão é igualmente maior assim como a seletividade ao F-Ac, que é praticamente o único produto observado conforme mostra na Figura 5.

\section{Efeito da quantidade de catalisador TBD}

A Figura 6 descreve os resultados obtidos variando-se as quantidades de catalisador TBD. A conversão de furfural aumenta com uma maior quantidade molar da base, como era de se esperar. Com $6 \mathrm{~mol} \%$ de TBD/mol de furfural observa-se $100 \%$ de conversão em menos de 1 h de reação. Este mesmo valor de conversão é alcançado

Tabela 1. Desempenho catalítico dos catalisadores PIP, APTES e TBD a $50{ }^{\circ} \mathrm{C}$ e $2 \mathrm{~h}$

\begin{tabular}{ccccc}
\hline Catalisador & $\begin{array}{c}\text { Conversão furfural } \\
{[\%]}\end{array}$ & $\begin{array}{c}\text { Seletividade F-OH } \\
{[\%]}\end{array}$ & $\begin{array}{c}\text { Seletividade F-Ac } \\
{[\%]}\end{array}$ & $\begin{array}{c}\text { F.T. } \\
{\left[\mathrm{mmol} \mathrm{F}^{-A c} \mathrm{mmol} \mathrm{cat}^{-1} \mathrm{~h}^{-1}\right]}\end{array}$ \\
\hline PIP & 2 & 35 & 65 & 0,08 \\
APTES & 30 & 45 & 55 & 0,99 \\
TBD & 84 & 27 & 73 & 3,67 \\
\hline
\end{tabular}




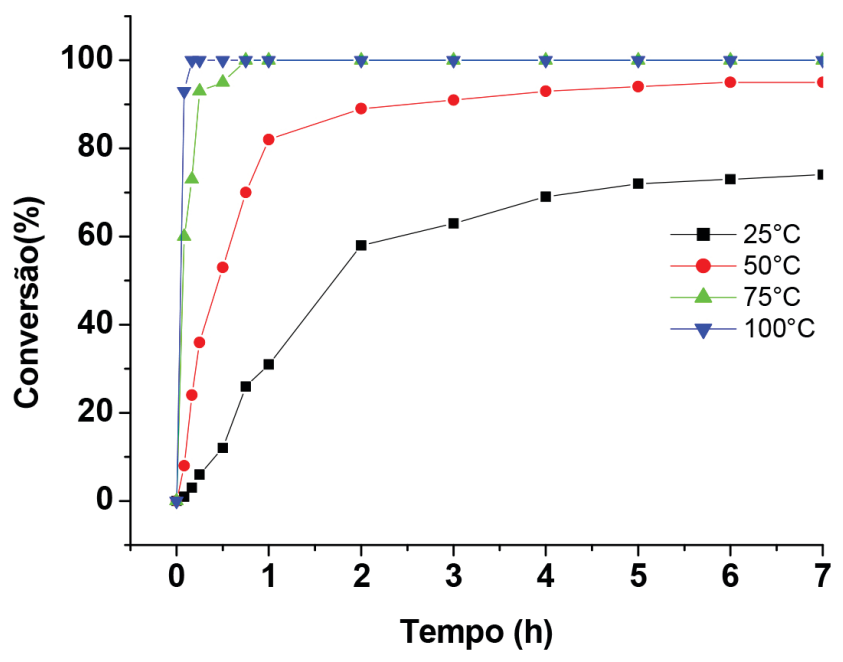

Figura 4. Influência da temperatura na conversão do furfural. Condições reacionais: 1,4 mol\% catalisador TBD/mol furfural, razão molar acetonal furfural igual 10:1; 7 h de reação

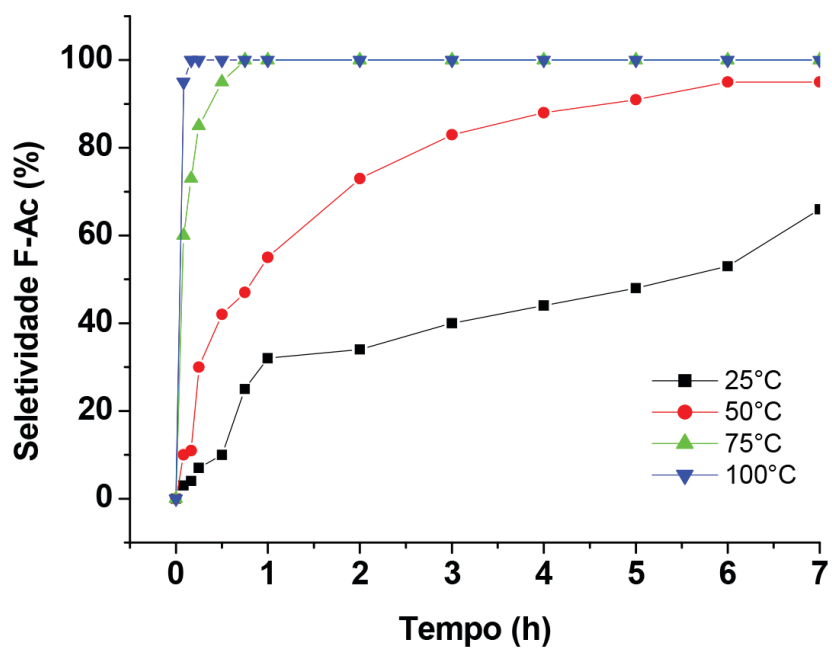

Figura 5. Influência da temperatura na seletividade do produto F-Ac. Condições reacionais: 1,4 mol\% catalisador TBD/mol furfural, razão molar acetona/furfural igual 10:1; 7 h de reação

em cerca de 2 h quando se usa $3 \mathrm{~mol} \%$ de TBD/mol de furfural. No valor de $1,4 \mathrm{~mol} \%$ de TBD/mol de furfural a conversão integral só é obtida após cerca de $7 \mathrm{~h}$; com o valor de $0,7 \mathrm{~mol}$ de TBD/mol de furfural a conversão atinge em torno de $40 \%$ após $7 \mathrm{~h}$.

A Figura 7 mostra a seletividade ao produto F-Ac. Nota-se que com a maior quantidade de base a seletividade chega a $100 \% \mathrm{em}$ menos de 1 h de reação. Para o valor de $3 \mathrm{~mol} \%$ de TBD/mol de furfural a seletividade de $100 \%$ é atingida entre 2 e 3 h. Nos outros dois casos, o intermediário F-OH é sempre observado no intervalo de tempo estudado. Para uma quantidade de 1,4 mol\% de TBD/mol de furfural a seletividade ao F-Ac é de $95 \%$ após 7 h, enquanto que para $0,7 \mathrm{~mol} \%$ de TBD/mol de furfural a seletividade a este produto é em torno de $20 \%$, após as mesmas $7 \mathrm{~h}$.

Os dados de conversão e seletividade mostram que é importante ajustar uma concentração de base ao meio reacional. Maiores quantidades levam, como esperado, a menores tempos para se atingir conversão e seletividade de $100 \%$. Todavia, uma maior quantidade de base incorre num maior custo operacional, já que a base TBD não é barata. Assim, uma quantidade entre 1,4 e 3 mol\% de TBD/mol de furfural parece a ideal para comprometer bons resultados de conversão e seletividade a um custo aceitável para o processo.

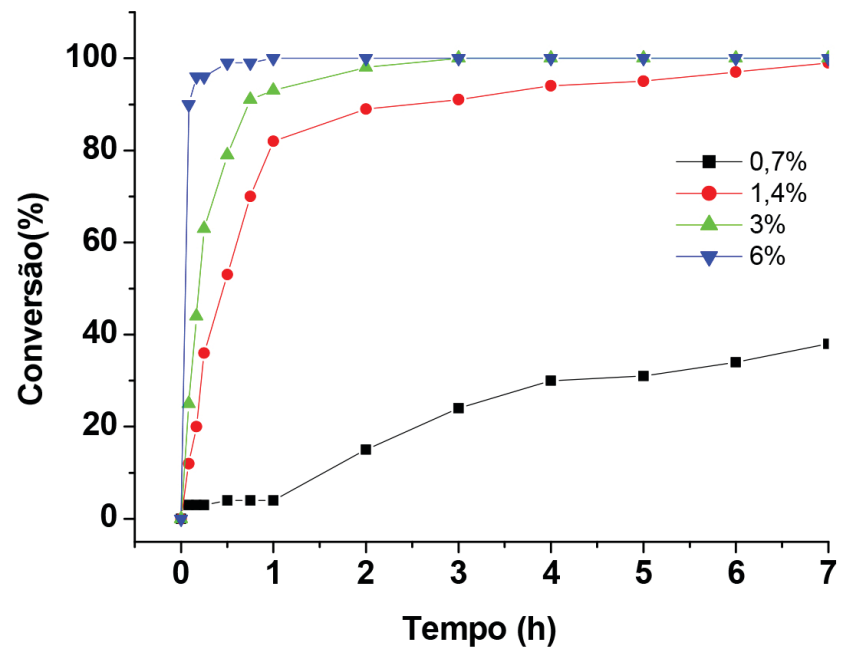

Figura 6. Efeito da quantidade de TBD na conversão de furfural. Condições reacionais: $50^{\circ} \mathrm{C}$, razão molar acetona/furfural igual 10:1 e $7 \mathrm{~h}$ de reação

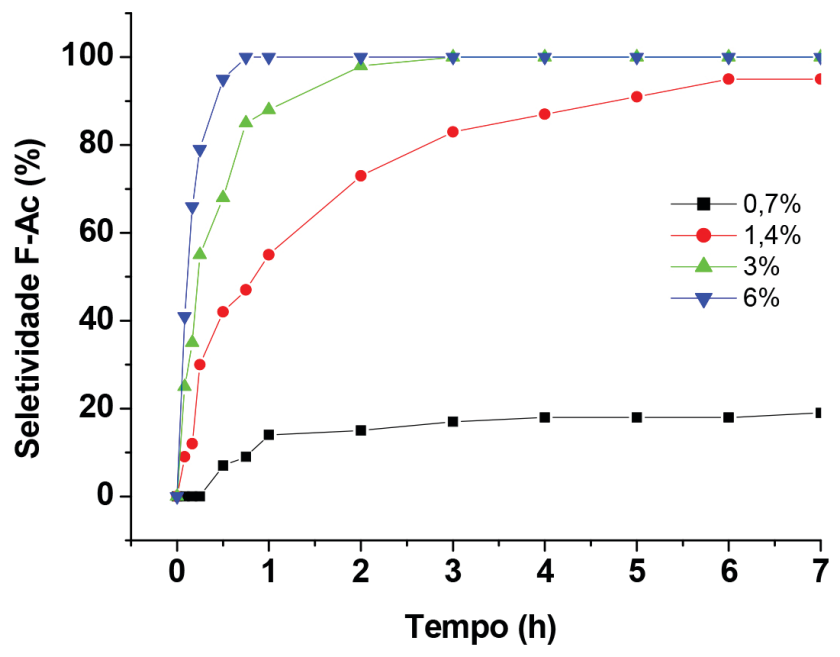

Figura 7. Efeito da quantidade de TBD na seletividade ao produto F-Ac ao longo do tempo de reação. Condições reacionais: $50{ }^{\circ} \mathrm{C}$, razão molar acetonal furfural igual 10:1 e $7 \mathrm{~h}$ de reação

\section{Efeito da razão molar acetona/furfural}

Na quarta série de experimentos, o objetivo foi comparar a atividade catalítica do TBD em diferentes razões molares acetona/furfural. As condições reacionais foram $50{ }^{\circ} \mathrm{C}$ em $6 \mathrm{~h}$ de reação. Em todos os experimentos houve somente a formação do intermediário F-OH e do produto F-Ac. A Tabela 2 mostra os resultados. É possível verificar que a conversão de furfural e seletividade ao F-Ac é crescente quando a razão molar acetona/furfural aumenta de 1:2 até 15:1.

A primeira etapa no mecanismo da condensação aldólica é a desprotonação da acetona para formação do enolato que, em seguida, ataca a molécula de furfural. A maior razão molar acetona/furfural favorece a reação, pois aumenta a concentração do enolato no meio reacional e, por conseguinte, a conversão do furfural. Dessa maneira, a taxa de reação depende da quantidade de sítios básicos e da formação de intermediários enolatos.

\section{COMPARAÇÃO ENTRE TBD E OUTROS CATALISADORES}

O catalisador TBD foi comparado com catalisadores heterogêneos, como óxidos mistos e hidrotalcitas. Os resultados estão representados 
Tabela 2. Efeito da razão molar acetona/furfural na reação em temperatura de $50{ }^{\circ} \mathrm{C}$ e $1,4 \mathrm{~mol} \%$ de $\mathrm{TBD} / \mathrm{mol}$ furfural

\begin{tabular}{|c|c|c|c|c|c|}
\hline $\begin{array}{l}\text { Razão molar acetona/ } \\
\text { furfural }\end{array}$ & $\begin{array}{l}\text { Tempo } \\
\text { (hora) }\end{array}$ & $\begin{array}{c}\text { Conversão } \\
(\%)\end{array}$ & $\begin{array}{l}\text { Seletividade } \\
\text { F-OH }(\%)\end{array}$ & $\begin{array}{l}\text { Seletividade } \\
\text { F-Ac }(\%)\end{array}$ & $\begin{array}{l}\text { Rendimento } \\
\text { F-Ac(\%) }\end{array}$ \\
\hline $1: 2$ & 6 & 76 & 20 & 80 & 61 \\
\hline 1:1 & 6 & 80 & 15 & 85 & 68 \\
\hline $5: 1$ & 6 & 82 & 15 & 85 & 70 \\
\hline $10: 1$ & 6 & 97 & 5 & 95 & 92 \\
\hline $15: 1$ & 6 & 98 & 4 & 96 & 94 \\
\hline
\end{tabular}

Tabela 3. Comparação entre catalisadores TBD, óxidos metálicos e hidrotalcitas

\begin{tabular}{|c|c|c|c|c|c|c|}
\hline Entrada & Catalisador & $\begin{array}{c}\% \text { mol catalisador/ } \\
\text { mol furfural }\end{array}$ & Temperatura $\left({ }^{\circ} \mathrm{C}\right)$ & Tempo (h) & $\begin{array}{l}\text { Conversão } \\
\text { Furfural (\%) }\end{array}$ & $\begin{array}{c}\text { Seletividade } \\
\text { F-Ac }(\%)\end{array}$ \\
\hline 1 & Hidrotalcita Co-Al ${ }^{\mathrm{a}, \mathrm{b}, 21}$ & - & 120 & 10 & 83 & 68 \\
\hline 2 & $\mathrm{MgO}-\mathrm{ZrO} 2^{\mathrm{c}, 20}$ & 4,5 & 50 & 24 & 81 & 14 \\
\hline 3 & $\mathrm{MgO}-\mathrm{Al}_{2} \mathrm{O}_{3}{ }^{\mathrm{c}, 20}$ & 4,5 & 50 & 24 & 64 & 31 \\
\hline 4 & $\mathrm{TBD}^{\mathrm{a}}$ & 6 & 50 & 1 & 100 & 100 \\
\hline 5 & $\mathrm{TBD}^{\mathrm{a}}$ & 3 & 50 & 3 & 100 & 100 \\
\hline 6 & $\mathrm{TBD}^{\mathrm{a}}$ & 1,4 & 50 & 7 & 99 & 95 \\
\hline
\end{tabular}

Condições reacionais: a) razão molar acetona/furfural igual 10:1; b) O \%mol para hidrotalcita não foi relatado porque o material não tem massa molecular correspondente e os autores não forneceram informações de medidas quantitativas de sítios básicos; c) razão molar acetona/furfural igual 1:1.

na Tabela 3. Nota-se que o TBD possui maior atividade e seletividade que os catalisadores básicos heterogêneos mais tradicionais. Os óxidos mistos de $\mathrm{MgO}-\mathrm{ZrO}_{2}$ (entrada 2) e $\mathrm{MgO}-\mathrm{Al}_{2} \mathrm{O}_{3}$ (entrada 3) apresentam conversões menores, assim como menor seletividade ao produto F-Ac, numa mesma temperatura reacional. Vale salientar que nestes experimentos a razão molar acetona/furfural foi de 1:1, mas o tempo foi significativamente maior, assim como a quantidade molar de catalisador. Já para a hidrotalcita de Co e Al, numa mesma razão molar de 10:1 de acetona/furfural, a conversão foi de $83 \%$, mas a $120^{\circ} \mathrm{C}$ e $10 \mathrm{~h}$ de reação. Estes dados mostram que o TBD tem grande potencial de ser utilizado como catalisador nesta reação, sobretudo se for ancorado em um suporte inorgânico, com vistas à reutilização do catalisador.

\section{CONCLUSÕES}

Três diferentes tipos de catalisadores básicos foram testados em reação de condensação aldólica entre a acetona e o furfural. O composto orgânico 1,5,7-Triazabiciclo[4.4.0]dec-5-eno (TBD) teve melhor atividade catalítica comparada à Piperazina (PIP) e ao 3-Aminopropil trietoxisilano (APTES), apresentando maior conversão de furfural, e seletividade ao 4-(2-Furil)-3-buten-2-ona. Este resultado está associado à maior basicidade do TBD em comparação com as outras aminas.

Experimentos de variação de condições reacionais mostraram que conversão de $100 \%$ pode ser alcançada em temperaturas de 75 ${ }^{\circ} \mathrm{C}$ em cerca de $1 \mathrm{~h}$ de reação. A quantidade molar de base em relação ao furfural, bem como a razão molar acetona/furfural, afetam significativamente a conversão e seletividade da reação. Um percentual de TBD de 3\% molar em relação ao furfural já permite obter 100\% de conversão e seletividade em $3 \mathrm{~h}$, enquanto uma razão molar de acetona/furfural de 10:1 parece ser a mais adequada para obtenção de alta conversão e seletividade.

\section{AGRADECIMENTOS}

Os autores agradecem as agências de fomento CAPES, CNPq e FAPERJ (E-26/202.741/2016) pelo suporte financeiro.

\section{REFERÊNCIAS}

1. Mota, J. A. J.; Monteiro, R. S.; Quim. Nova 2013, 36, 1483.

2. Suarez, P. A. Z.; Santos, A. L. F.; Rodrigues, J. P.; Alves, M. B.; Quim. Nova 2009, 32, 768.

3. Rodrigues, J. A. R.; Quim. Nova 2011, 34, 1242.

4. Azadi, P.; Malina, R.; Barrett, S. R. H.; Kraft, M.; Renewable Sustainable Energy Rev. 2017, 76, 1479.

5. Dabdoub, M. J.; Bronzel, J. L.; Quim. Nova 2009, 32, 776.

6. Lima, P. S.; Ferreira, L. A.; Freitas, L. H.; Rheinheimer, M. W.; Sokolovicz, Y. C. A.; Schrekker, H. S.; J. Braz. Chem. Soc. 2014, 25, 2266.

7. Diederichs, G. W.; Mandegari, M. A.; Farzad, S.; Görgens, J. F.; Bioresour. Technol. 2016, 216, 331.

8. Silva, N. L.; Maciel, M. R. W.; Maciel Filho, R.; Batistella, C. B.; PI0803465-6, 2008.

9. Chiaramonti, D.; Prussi, M.; Buffi, M.; Tacconi, D.; Appl. Energy 2014, 136, 767.

10. Cremonez, P. A.; Feroldi, M.; Araújo, A. V.; Borges, M. N.; Meier, T. W.; Feiden, A.; Teleken, J. G.; Renewable Sustainable Energy Rev. 2015, 43, 1063.

11. West, R. W.; Liu, Z. Y.; Dumesic, J. A.; ChemSusChem. 2008, 1, 417.

12. Mccall, M. J.; Kocal, J. A.; Bhattacharyya, A.; Kalnes, T. N.; Brandvold, T. A.; US8039682B2, 2011.

13. Maciel Filho, R.; Batistella, C.; Maciel, Wolf, M. R.; Silva, N. L.; AS O667963097BR - C1, 2012.

14. Fakhfakh, N.; Cognet, P.; Cabassud, M.; Lucchese, Y.; Los Rios, M. D.; Chem. Eng. Process 2008, 4, 349.

15. Sun, Y.; Cheng, J.; Bioresour. Technol. 2002, 83, 1.

16. Huber, G. W.; Dumesic, J. A.; Catal. Today. 2006, 111, 119.

17. Shreve, R. N.; Brink, J. A. J.; Chemical Process Industries, $4^{\text {th }}$ ed., McGraw-Hill: Washington, 1997.

18. Huber, G. W.; Dumesic, J. A.; Science 2005, 308, 1446.

19. West, R. M.; Liu, Z. Y.; Peter, M.; Gartner, C. A.; Dumesic, J. A.; J. Mol. Catal. A 2008, 296, 18.

20. Faba, L.; Diaz, E.; Ordonez, S.; Biomass Bioenergy 2013, 56, 592.

21. Xu, C.; Liu, Y. G. X.; Xin, R.; Wang, Z.; RSC Adv. 2013, 3, 793. 
22. Kikhtyanin, O.; Chlubná, P.; Jindrová, T.; Kubicka, D.; Dalton Trans. 2014, 43, 10628.

23. Kikhtyanina, O.; Kelbichová, V.; Vitvarová, D.; Kubu, M.; Kubicka, D.; Catal. Today 2014, 227, 154.

24. Schroeder, G.; Luska, B.; Jarczewski, A. Nowak-Wydra, B.; Brzezinski, B.; J. Mol. Struct. 1995, 344, 77.

25. Simoni, D.; Rondanin, R.; Morini, M.; Baruchello, R.; Invidata, F. P.; Tetrahedron Lett. 2000, 41, 1607.

26. Horvath, A.; Tetrahedron Lett. 1996, 37, 4423.

27. Simoni, D.; Rossi, M.; Rondanin, R.; Mazzali, A.; Baruchello, R.; Malagutti, C.; Roberti, M.; Invidata, F. P.; Org. Lett. 2000, 2, 3765.

28. Cota, I.; Medina, F.; Sueiras, J.; Tichit, D.; Tetrahedron Lett. 2011, 52, 385 .
29. Srivastava, R.; J. Mol. Catal. A 2007, 264, 146.

30. De Lima, A. L.; Mbengue, A.; Gil, R. A. S.; Ronconi, C. M.; Mota, C. J. A.; Catal. Today 2014, 226, 210.

31. Rana, S.; Jonnalagadda, S. B.; Catal. Commun. 2017, 92, 31.

32. Shanmuganathan, S.; Greiner, L.; de María, P. D.; Tetrahedron Lett. 2010, 51, 6670 .

33. Das,S. K. ; Wang, X.; Ostwal, M. M.; Zhao, Y. F.; Han, Y.; Lai, Z.; Chem. Eng Sci. 2016, 145, 21.

34. Vilarrasa-García, E.; Cecilia, J. A.; Azevedo, D.C.S.; Cavalcante Jr., C.L.; Rodríguez-Castellon, E.; Microporous Mesoporous Mater. 2017, $249,25$. 